\title{
PALINDROMIC WORD PUZZLES
}

\author{
Thinh D. Nguyen \\ Email: kosmofarmer@yandex.com
}

\begin{abstract}
We show that it is NP-complete to determine if a given set of strings can be arranged to form a palindrome.
\end{abstract}

\section{PROBLEM STATEMENT}

We give an example: The strings \{"German" "man" "am" "am" "I" "I" "regal" "a"\} canbe arranged into "I man am regal a German am I". The strings \{"bat" "bat"\} cannot be arranged into a palindrome, because we are not allowed to change the order of characters within strings ("bat tab" is not allowed).

This reminds us of the problem of determining if a set of jigsaw puzzle pieces can all fit together, which is NP-complete.

\section{PROOF OF HARDNESS}

Setup of proof: We show that the problem is NP-hard by a reduction from 3-partition.

Suppose that $m$ and $n$ are integers, and $n$ is divisible by 3 . Suppose that $l_{1}, l_{2}, l_{3}, \ldots, l_{n}$ are integers where $m / 4<l_{i}<m / 2$ for all $i$. Then determining if $l_{1}, l_{2}, l_{3}, \ldots, l_{n}$ can be arranged into triplets $l_{i}, l_{j}, l_{k}$ such that $l_{i}+l_{j}+l_{k}=m$ is NP-complete. We show that for every instance of this problem, there exists an instance of the previously described palindrome problem such that a YES for 3-partition means a YES for the palindrome problem, and a NO for 3-partition means a NO for the palindrome problem.

To make such an instance, we name one string $\mathbf{S}$ and let it equal "aaa...aaa,aaa...aaa,.., aaa... $a a a^{\prime \prime}$, which is a string that consists of $m a^{\prime}$ s, followed by a comma, followed by $m a^{\prime}$ s, followed by a comma, and continuing this pattern until it ends with $m a$ 's. This string contains $n-1$ commas. In other words, the string is $n$ groups of $m$-long strings of $a$ 's, separated by commas.

Example: $m=6, n=4$ then $\mathbf{S}=$ "aaaaaa,aaaaaa,aaaaaa,aaaaaa"

This is 4 groups of 6-long strings of $a$ 's, separated by commas.

Then we define strings $s_{i}$, for $1 \leq i \leq n$, to contain a number of $a$ 's equal to the value of $l_{i}$.

We then define strings $b_{i}=$ "," for $1 \leq i \leq n-1$.

In order to arrange strings $\mathbf{S}, s_{i}$, and $b_{i}$ (for all $i$ that these strings are defined) into a palindrome, we have to place the long string $\mathbf{S}$ on one side, and try to form triplets from the strings $s_{i}$ so that each triplet has exactly $m a$ 's in it. Then we just put a comma between each triplet. So, this specific type of the palindrome problem is exactly the same as a 3-partition problem.

\section{CONCLUSION}


Garey and Johnson [1] shapes their theory based on previous primal works of Cook, Levin and Karp. Johnson [2] moves on with the guide to this theory. As long as we study a mathematical conjecture, we should encourage ourselves to have moderate amount of labour hours on popular maths books like these.

Then, reading some articles on theory of computing like [3] is a good practice. Having all those done, we could think of the ultimate final for all mathematics sciences.

\section{REFERENCE}

1. Michael R. Garey, David S. Johnson, Computers and Intractability: A Guide to the Theory of NP-Completeness

2. David S. Johnson, The NP-Comple teness Column: An Ongoing Guide

3. Phan Dinh Dieu, Le Cong Thanh, Le Tuan Hoa, Ave rage Polynomial Time Complexity of Some NP-Complete Problems, Theor. Comput. Sci. 46(3): 219-237 (1986) 